\title{
Außerordentliche Tagung der Ophthalmologischen Gesellschaft in Wien vom 4. bis 6. August 1921
}

Der Einladung der Wiener Ophthalmologischen Gesellschaft war eine große Anzahl reichsdeutscher Kollegen gefolgt. Durch starke Beteiligung auch der Augenärzte DeutschÖsterreichs, ÜDgarns und der Eachbarländer, sowie des neutralen Auslandes war ein festlicher Rahmen für die Ehrung des Altmeisters Ernst Fuchs geschaffen. Die Tagung fand in den Räumen der 1. Univ.-Augenklinik statt, die, im Jahre 1812 begründet, wohl die älteste Augenklinik der "Welt ist und uns alien ehrwürdig erscheint durch ihre großen Traditionen.

Den Inhalt der Vorträge und Demonstrationen gibt in Kürze der Bericht wieder, den Lauber in dieser Zeitschrift erstatten wird.

Allgemein war wohl das Bedauern darüber, daß die Gastgeber selbst, die Mitglieder der Wiener Ophthalmologischen Gesellschaft, sich mit ihren Vorträgen an den Schluß des für die knapp bemessene Zeit überreichen Programme gesetzt hatten und nicht mehr zu Wort kamen. Wir hoffen aof Abdruck der beabsichtigten Mitteilungen im offiziellen Bericht der Tagung.

x) Der offizielle Bericht erscheint im Verlag von S. Karger.

in Wien 4. bis 6. August 1921.

117

Von allgemeinsfcem Interesse waren die Vorträge und die Aussprache über das Glaukom. Die Referate liierzu wurden von Uhthoff und Wessely erstattet

Der Vortrag von Uhthoff (klinischer Teil) stellte monumental die jahr-zehntelangen Erfahrungeu der Breslauer Klinik und die besonderen Beobach-tuugen des Redners in seiner ausgedehnten Privattätigkeit zusammen, in welcken die Verhältnisse beitn Glaukom nach Art und Lebensweise der Patienten öfter abweichend liegen.

Das Refßrat von Wessely (anatomischer und theoretischer Teil), mit souveräuer Beherrschung des Stoffes zusammengefaßt, erschien wohl alien Hörern als der Höhepunkt der

wissenschaftlichen Tagung.

Beide Vorträge und die Aussprache brachten uns aber auch. zum Be-waßtsein, wie vieles wir vom Glaukom noch nicht wissen und wie wenig wir einzelnen Fallen gegenüber therapeutisch zielbewußt vorgehen können. TJnddarin liegt wohl der Hauptreiz dieser Erörterungen: daß sie weiter anregen zur exakten Fragestellung und zur Durchforschung der schwierigen Probleme, die noch vor uns liegen und der Lösung warten.

Die allgemeine Organisation des Kongresses, als dessen Schriftführer L'auber mit größter Umsicht waltete, war vorbildlich; Prof. J. Meller, der als Hausherr der Klinik die Gäste begrüßte, ihnen nach Wunsch Einblick in die Einrichtungen des Hauses gab und interessante Fälle vorstellen ließ, wurde hierbei von seinen Assistenten, insbesondere von Herrn Dr. ßachstez, bestens unterstützt, der unermüdllch um jeden besorgt war.

Nach der Arbeit des Tages war auch für die Erholung, für kollegialen Zusammenhalt und persönliches Kennenlernen der Gäste untereinander reiche Vorsorge getroffen. Niemand wird an den Kongreß zurückdenken können, ohne zugleich mit Dank und Bewunderung der Fürsorge 
und Or-ganisationskunst des Koilegen Richard Kramer siçh zu erinnern, der alles beschaffte, was dem einzelnen erwünscht und, weil rationiert, für gewöhn-liche Sterbliche in Wien derzeit nicht leicht erreichbar war: Unterkunft, Verpflegung, Rauchmaterial, Aussichtswagen der Straßenbahn für die Aus-flüge, Reisekarten für rechtzeitige Heimkehr, eine für die Fremden uner-wartete besondere Schwierigkeit. Die Herren Assistenten der 1. und 2. Universitäts - Augenklinik unterstützten Dr. Kramer hilfreichst, wo seine Unermüdlichkeit nicht ausreichte. Der erste Abend führte die Kongreßteilnehmer zu den Anhöhen um Wien, von denen aus ein prachtvoller Buck auf die schöne alte Kaiserstadt sich bietet. Im „Krapfenwaldl” wurde gespeist und geplaudert, und die Sorgen, die uns aïle in dieser trüben Zeit bedrücken, schwanden auf einige Stunden, die auch in alter Zeit kaum behaglicher hätten gestaltet werden können. Hofrat Prof. Dimmer gedachte der Bedeatung der Veranstaltung, die zum ersteu Male Gäste der Ophthalmologischen Gesellschaft nach Wien geführt habe. Er feierte ganz schlicht auch Ernst Fuclis, der zu aller Freude in voller Frische anwesend war. Führende Ophthalmologen der Lander, die vertreten waren, schlossen sich ihm an. ííiemand zweifelte, daß Wien ein hohes geistiges Kulturzentrum wie bisher trotz aller Note der Gegenwart auch ferner bleiben werde. Der frische Geist, der iu der Ophthalmologischen Gesellschaft Wiens alien Augen erkenntlich lebt, ist speziell uns Augenärzten Bürge dafür, daß die wissenschaftliche Augenheilkunde in "Wien auch für die Zukunft wohlgeborgen ist und Früchte reifen lassen wird, die der Gesamtheit zu-gute kommen.

Am zweiten und dritten Abend verteilten sich die Teilnehmer zwang-los in kleinere Gruppen zu verschiedenen Veranstaltungen: Wanderungen in die Umgebung, zum „Heurigen”, zur “Wiener Operette. $\mathrm{Zu}$ allem fanden sich freundliche und kundige Führer.

Der Sonntag war dem gemeinsamen Ausflug nach dem Semmering, dem bekannten reizvollsten Pankt in Wiens Umgebung, gewidmet. Es hätte kein schönerer Ausklang für die anregenden, aber durch die Hitze des Sommers und die Eatfernungen in der großen Stadt auch anstrengenden $11 \mathrm{~g}$ Literatur-Verzeichnís.

Tage gefunden werden können als diese Eahrt zur reinen Höhe der Berg-welt. JDer Tag verlief in voller B $1 / 8$ rmonie. Der Dank, den berafene $\mathrm{P}^{1} / 8$ ednerden G-astgebern aussprachen, kam vom Herzen. Ein neues Band derEreundschaft ist zwischen den österreichischen Kollegen und iliren Gästengeschlungen und reiche Anregungen sind ausgestreut. Sie werden auchiür die Wissenschaft von Nutzen tsein. Junius (Bonn). Litepatur-Verzeichnis.

Anatomie - Physiologie - Pathologie. Angelucci, A., Le origini posifciviste della cosoienza estetica. Arch, di Ottalm.

Bd. 28, H. 1-2. Bab, Werner, Die Ursachen der Kriegsblindheit. Berl. klin. Woch. Nr. 20. Babonneix, L., Spécificité on non-spécificité du signe d'Argyll-Robertson.

Gaz. des hôpit. Nr. 25. Bailey, John H., Traumatic myopia. Amer. Journ. of ophthalm. Mai 1921. Baldino, S., Bapporto dell'acuitá visiva con la grandezza del globo ocuíare.

Arch, di Ottalm. Bd. 27, Nr. 9-11. Brown, Edw. J., Corneal and scleral measurements of intraocular tension with

Brown Tonometer. Amer. Journ. of ophthalm. Mai 1921. Butler, Harrison T., Sloughing in Graves's Disease and in Disseminated Sclerosis. Brit. Journ. of ophthalm. Juli 1921. Campbell, Don M. u., John M. Carter, Injuries to the eye, with report of cases.

Amer. Journ. of ophthalm. Mai 1921. Cantonnet, A., L'examen de Гocil a la lumière. Pr. méd. Nr. 34. Derby, George S., Intraocular foreign body. A surgical emergency. Amer. 
journ. of ophthalm. Mai 1921. Erggelet, H., Versuche zur beidäugigen Tiefenwahrnehmung bei hoher Ungleich-

sichtigkeit. Klin. Monatsbl. f. Augenh. Mai 192]. Ferrari, G., Le lesioni del fondo ocuiare nelP encefalite letargica. Arch, di Ottalm.

Bd. 27, Nr. 12. Fischer, Max Heinrich, Messende Untersuchungen über das scheinbare

Gleichhoch, Geradevorne und Stirngleich. (Ein Beitrag zur Lehre vom

funktionellen Koordinatensystem des Gesichtsraumes.) Arch. f. Physiol.

Bd. 188, H. 4-6. Fleischer, B., Beiträge zur Vererbung von Augenkrankheit en. Klin. Monatsbl. f. Augenh. Mai 1921. Friedenwald, Harry, Ocular Conditions Associated with Arthritis. Amer. Journ.

of ophthalm. Juni 1921. Fuchs, Ernst, Lehrbuch der Augenheilkunde. 13. Aufl. Bearbeitet von M. Salz-

mann. Leipzig-W’ien, ¥. Deuticke. Gaudissart, P., The Ocular Blood Tension. Amer. Journ. of Ophthalm. Juli 1921. Gilbert, W., Über Pigmentanomalien des Auges. Arch, für Augenh. Bd. 88, H. 3 u. 4. Groethuysen, G., Über die Beziehungen zwischen motorischer und optischer 